PROCEEDINGS OF THE

AMERICAN MATHEMATICAL SOCIETY

Volume 136, Number 9, September 2008, Pages 3177-3183

S 0002-9939(08)09298-8

Article electronically published on May 1, 2008

\title{
GENERALIZED INVERSES AND DOUGLAS EQUATIONS
}

\author{
M. LAURA ARIAS, GUSTAVO CORACH, AND M. CELESTE GONZALEZ \\ (Communicated by N. Tomczak-Jaegermann)
}

\begin{abstract}
In this paper we study the general concept of reduced solution for a Douglas type equation and we parametrize these solutions using generalized inverses. On the other hand, we characterize different sorts of generalized inverses by means of solutions of Douglas type equations.
\end{abstract}

\section{INTRODUCTION}

This paper is devoted to making explicit the relationship between the notions of Douglas solutions of operator equations like $A X=B$ and that of generalized inverses of bounded linear operators with closed range. These equations arise in many problems of engineering, physics, and statistics. The following well-known theorem, due to R. G. Douglas [4] (see also [5]), provides equivalent conditions for the existence of solutions.

Theorem 1.1. Let $\mathcal{H}, \mathcal{K}, \mathcal{G}$ be Hilbert spaces, $A \in L(\mathcal{H}, \mathcal{K})$ and $B \in L(\mathcal{G}, \mathcal{K})$. The following conditions are equivalent:

(1) $R(B) \subseteq R(A)$.

(2) There is a positive number $\lambda$ such that $B B^{*} \leq \lambda A A^{*}$.

(3) There exists $C \in L(\mathcal{G}, \mathcal{H})$ such that $A C=B$.

If one of these conditions holds, then there is a unique operator $D \in L(\mathcal{G}, \mathcal{H})$ such that $A D=B$ and $R(D) \subseteq \overline{R\left(A^{*}\right)}$. Moreover, $\|D\|^{2}=\inf \left\{\lambda>0: B B^{*} \leq \lambda A A^{*}\right\}$. We shall call $D$ the Douglas solution of $A X=B$.

We are interested in methods for finding the solutions $C$ and $D$. For this, the concept of a generalized inverse of an operator is very helpful. An operator $A^{\prime} \in$ $L(\mathcal{K}, \mathcal{H})$ is said to be a generalized inverse of an operator $A \in L(\mathcal{H}, \mathcal{K})$ if

$$
\text { 1. } A A^{\prime} A=A \text {. }
$$

$A \in L(\mathcal{H}, \mathcal{K})$ has a generalized inverse if and only if $R(A)$ is closed. In fact, if $A^{\prime}$ is a generalized inverse of $A$, then $A A^{\prime} A A^{\prime}=A A^{\prime}$, i.e., $A A^{\prime}$ is a projection onto the closed subspace $R\left(A A^{\prime}\right)$. Furthermore, $R(A)=R\left(A A^{\prime} A\right) \subseteq R\left(A A^{\prime}\right) \subseteq R(A)$, so $R(A)=R\left(A A^{\prime}\right)$, and therefore it is closed. Conversely, if $R(A)$ is closed, then $P_{R(A)}$ is a bounded linear operator and, by the Douglas theorem, the equation

Received by the editors April 18, 2007, and, in revised form, July 13, 2007.

2000 Mathematics Subject Classification. Primary 47A50, 15A09.

Key words and phrases. Douglas equation, generalized inverse, oblique projections.

The authors were supported in part by UBACYT I030, CONICET PIP 5272.

(C)2008 American Mathematical Society Reverts to public domain 28 years from publication 
$A X=P_{R(A)}$ has a solution; i.e., there exists $A^{\prime} \in L(\mathcal{K}, \mathcal{H})$ such that $A A^{\prime}=P_{R(A)}$. Then $A A^{\prime} A=A$ and so $A$ has a generalized inverse.

As a consequence, if $R(B) \subseteq R(A)$ and $R(A)$ is closed, then $\left\{A^{\prime} B: A A^{\prime} A=A\right\}$ is a set of solutions of the equation $A X=B$. In fact, $A\left(A^{\prime} B\right)=Q B=B$, because $Q=A A^{\prime}$ is a projection onto $R(A)$.

If $A \in L(\mathcal{H}, \mathcal{K})$ has closed range, then it has a unique generalized inverse $A^{\prime}$ in $L(\mathcal{K}, \mathcal{H})$ which also verifies:

$$
\begin{aligned}
& \text { 2. } A^{\prime} A A^{\prime}=A^{\prime}, \\
& \text { 3. }\left(A A^{\prime}\right)^{*}=A A^{\prime}, \\
& \text { 4. }\left(A^{\prime} A\right)^{*}=A^{\prime} A .
\end{aligned}
$$

This distinguished generalized inverse is called the Moore-Penrose (generalized) inverse of $A$ and is denoted by $A^{\dagger}$. This result is due to E. H. Moore [9] and R. Penrose 11], 12]. For excellent expositions of these matters the reader is referred to the books [10] and [1] among many other sources. If $R(B) \subseteq R(A)$ and $R(A)$ is closed, then $A^{\dagger} B$ is the Douglas solution of $A X=B$. This assertion is the starting point of this paper. We denote $A[i], A[i, j], A[i, j, k], A[i, j, k, l]$ as the sets of operators $X$ in $L(\mathcal{K}, \mathcal{H})$ that satisfy equations $\{i\},\{i, j\},\{i, j, k\}$ and $\{i, j, k, l\}$, respectively, for $i, j, k, l=1,2,3,4$. Then, by the result of Moore and Penrose, $A[1,2,3,4]=\left\{A^{\dagger}\right\}$. As a byproduct of our approach we show a very short proof of the existence and uniqueness of the Moore-Penrose inverse of a matrix $A$.

In section 2 we introduce the notion of reduced solution which is a generalization of the concept of Douglas solution. We characterize the set of these solutions and we give a parametrization of this set using Douglas solutions.

In section 3 we establish the relationship between the sets $A[1], A[1, i], A[1, i, j]$ and $A[1, i, j, k]$ and different Douglas type equations.

Finally, we collect in section 4 some results on the continuity of the mapping $(A, B) \rightarrow$ Douglas solution of $A X=B$.

We conclude this introduction by fixing notation and terminology. Throughout the paper $\mathcal{H}, \mathcal{K}, \mathcal{G}$ denote complex Hilbert spaces, $L(\mathcal{H}, \mathcal{K})$ the space of bounded linear operators from $\mathcal{H}$ to $\mathcal{K}$, and $C R(\mathcal{H}, \mathcal{K})$ the subset of $L(\mathcal{H}, \mathcal{K})$ of all operators with closed range. The range of an operator $A$ is denoted by $R(A)$ and its nullspace by $N(A)$. We denote by $\mathcal{Q}$ the set of projections of $L(\mathcal{H})$, i.e., $\mathcal{Q}=$ $\left\{Q \in L(\mathcal{H}): Q^{2}=Q\right\}$. Fixed a closed subspace $\mathcal{S}$ of $\mathcal{H}, \mathcal{Q}_{\mathcal{S}}$ denotes the set of projections with range $\mathcal{S}$ and $P_{\mathcal{S}}$ denotes the orthogonal projection of $\mathcal{H}$ onto $\mathcal{S}$. The direct sum between two closed subspaces $\mathcal{S}, \mathcal{T}$ is denoted $\mathcal{S}+\mathcal{T}$. Observe that the existence of a decomposition $\mathcal{H}=\mathcal{S}+\mathcal{T}$ is equivalent to the existence of a unique $Q \in \mathcal{Q}_{\mathcal{S}}$ such that $N(Q)=\mathcal{T}$, where $Q$ is usually denoted by $Q_{\mathcal{S} / / \mathcal{T}}$.

The next propositions will be used in this paper. See [7] and [3], respectively, for the proofs.

Proposition 1.2. If $\mathcal{S}$ and $\mathcal{T}$ are closed subspaces of $\mathcal{H}$ and $\mathcal{S}+\mathcal{T}$ is closed, then

$$
(\mathcal{S} \cap \mathcal{T})^{\perp}=\mathcal{S}^{\perp}+\mathcal{T}^{\perp} .
$$

In particular, $\mathcal{S}^{\perp}+\mathcal{T}^{\perp}$ is closed. Consequently, $\mathcal{S}+\mathcal{T}$ is closed if and only if $\mathcal{S}^{\perp}+\mathcal{T}^{\perp}$ is closed.

Proposition 1.3. Let $\mathcal{S}$ and $\mathcal{T}$ be two closed subspace of $\mathcal{H}$. Then, $\mathcal{H}=\mathcal{S}+\mathcal{T}$ if and only if $\mathcal{H}=\mathcal{S}^{\perp}+\mathcal{T}^{\perp}$. 
Proposition 1.4. Let $A \in L(\mathcal{H}, \mathcal{K})$ and $B \in L(\mathcal{G}, \mathcal{H})$ with closed ranges. Then $A B$ has closed range if and only if $N(A)+R(B)$ is closed.

\section{Reduced SOLUtions}

The first part of the following theorem is a generalization of the Douglas theorem. We include the proof, which is similar to Douglas, original proof, for the reader's convenience. The second part provides a characterization of the generalized reduced solutions in terms of generalized inverses.

Theorem 2.1. Let $A \in L(\mathcal{H}, \mathcal{K})$ and $B \in L(\mathcal{G}, \mathcal{K})$ be such that $R(B) \subseteq R(A)$ and let $\mathcal{M}$ be a closed subspace of $\mathcal{H}$ such that $N(A)+\mathcal{M}=\mathcal{H}$. Then there exists a unique solution $X_{\mathcal{M}}$ of the equation $A X=B$ such that $R\left(X_{\mathcal{M}}\right) \subseteq \mathcal{M}$. The operator $X_{\mathcal{M}}$ will be called the reduced solution of the equation $A X=B$ for the subspace $\mathcal{M}$. Moreover, if $A \in C R(\mathcal{H}, \mathcal{K})$ and $Y \in L(\mathcal{G}, \mathcal{H})$, then $Y$ is the reduced solution of $A X=B$ for $\mathcal{M}$ if and only if $Y=A^{\prime} B$ for some $A^{\prime} \in A[1,2]$.

Proof. Since $R(B) \subseteq R(A)$, for each $x \in \mathcal{G}$ there exists $x_{\mathcal{M}} \in \mathcal{M}$ such that $B x=$ $A x_{\mathcal{M}}$. This $x_{\mathcal{M}}$ is unique: in fact, if there exists $\tilde{x}_{\mathcal{M}} \in \mathcal{M}$ such that $B x=A \tilde{x}_{\mathcal{M}}$, then $A\left(x_{\mathcal{M}}-\tilde{x}_{\mathcal{M}}\right)=0$. Thus, $x_{\mathcal{M}}-\tilde{x}_{\mathcal{M}} \in N(A) \cap \mathcal{M}=\{0\}$, and so $x_{\mathcal{M}}=\tilde{x}_{\mathcal{M}}$, as claimed. Therefore, the map $X_{\mathcal{M}}: \mathcal{G} \longrightarrow \mathcal{H}$ such that $X_{\mathcal{M}} x=x_{\mathcal{M}}$ is well defined and is linear. Moreover, $X_{\mathcal{M}} \in L(\mathcal{G}, \mathcal{H})$ because its graph, denoted by $\Gamma_{X_{\mathcal{M}}}$, is closed. In fact, let $\left(x_{n}, x_{\mathcal{M}_{n}}\right) \in \Gamma_{X_{\mathcal{M}}}$ such that $x_{n} \underset{n \rightarrow \infty}{\longrightarrow} x$ and $x_{\mathcal{M}_{n}} \underset{n \rightarrow \infty}{\longrightarrow} x_{\mathcal{M}}$; then, $A x_{\mathcal{M}}=\lim _{n \rightarrow \infty} A x_{\mathcal{M} n}=\lim _{n \rightarrow \infty} B x_{n}=B x$ and, so, $\left(x, x_{\mathcal{M}}\right) \in \Gamma_{X_{\mathcal{M}}}$. By the definition of the operator $X_{\mathcal{M}}$ it holds that $A X_{\mathcal{M}}=B$ and $R\left(X_{\mathcal{M}}\right) \subseteq \mathcal{M}$. It only remains to prove the uniqueness of $X_{\mathcal{M}}$ :

Let suppose that there exists $D \in L(\mathcal{G}, \mathcal{H})$ such that $A D=B$ and $R(D) \subseteq \mathcal{M}$. Then, $B^{*}=D^{*} A^{*}=X_{\mathcal{M}}^{*} A^{*}$, i.e., $\left(D^{*}-X_{\mathcal{M}}^{*}\right) A^{*}=0$. This gives $D^{*}=X_{\mathcal{M}}^{*}$ in $N(A)^{\perp}$. Now, since $\mathcal{H}=N(A)+\mathcal{M}=N(A)^{\perp} \dot{+} \mathcal{M}^{\perp}$, it remains to prove that $X_{\mathcal{M}}^{*}$ and $D^{*}$ coincide on $\mathcal{M}^{\perp}$. Let $z \in \mathcal{M}^{\perp}$; since $\mathcal{M}^{\perp} \subseteq R\left(X_{\mathcal{M}}\right)^{\perp}=N\left(X_{\mathcal{M}}^{*}\right)$ and $R(D)^{\perp}=N\left(D^{*}\right)$, then $X_{\mathcal{M}}^{*} z=D^{*} z=0$, and so $X_{\mathcal{M}}=D$ as desired.

Now consider $A \in C R(\mathcal{H}, \mathcal{K})$. Let $X_{\mathcal{M}}$ be the reduced solution of the equation $A X=B$ for some complement $\mathcal{M}$ of $N(A)$. We shall to prove that there exists $A^{\prime} \in A[1,2]$ such that $X_{\mathcal{M}}=A^{\prime} B$. Now, since $N(A)+\mathcal{M}=\mathcal{H}$, we can take the unique $Q \in \mathcal{Q}_{\mathcal{M}}$ such that $N(Q)=N(A)$. On the other hand, let $Q^{\prime} \in \mathcal{Q}_{R(A)}$. Then, $A^{\prime}=Q A^{\dagger} Q^{\prime} \in A[1,2]$ and $A^{\prime} B=A^{\prime} A X_{\mathcal{M}}=Q X_{\mathcal{M}}=X_{\mathcal{M}}$. Conversely, let $A^{\prime} \in A[1,2]$; then $A A^{\prime} \in \mathcal{Q}_{R(A)}$ and $A^{\prime} A \in \mathcal{Q}_{R\left(A^{\prime}\right)}$. Therefore, $A A^{\prime} B=B$, i.e., $A^{\prime} B$ is a solution of the equation $A X=B$. Let us see that there exists a closed subspace $\mathcal{M}$ of $\mathcal{H}$ such that $R\left(A^{\prime} B\right) \subseteq \mathcal{M}$ and $N(A)+\mathcal{M}=\mathcal{H}$. First, observe that since $A A^{\prime}$ has closed range, then, by Proposition 1.4 $R\left(A^{\prime}\right)+N(A)$ is closed. Furthermore, $N(A) \cap R\left(A^{\prime}\right)=\{0\}$. In fact, if $x \in N(A) \cap R\left(A^{\prime}\right)$, then $A x=0$ and so $x=A^{\prime} A x=0$. Thus, $\mathcal{S}=R\left(A^{\prime}\right) \dot{+} N(A)$ is closed. Now, consider $\mathcal{M}=R\left(A^{\prime}\right)+\mathcal{S}^{\perp}$. Then, as $R\left(A^{\prime}\right)^{\perp}+\mathcal{S}=\mathcal{H}$, by Proposition 1.2, $\mathcal{M}$ is closed. On the other hand, $\mathcal{M} \cap N(A)=\{0\}$ since $N(A) \cap R\left(A^{\prime}\right)=\{0\}$ and $N(A) \subseteq \mathcal{S}$. Then $\mathcal{M} \dot{+} N(A)=R\left(A^{\prime}\right)+\mathcal{S}^{\perp} \dot{+} N(A)=\mathcal{S}+\mathcal{S}^{\perp}=\mathcal{H}$. Therefore, $\mathcal{M}$ verifies the desired requirement and so $A^{\prime} B=X_{\mathcal{M}}$.

Corollary 2.2. If $A \in C R(\mathcal{H}, \mathcal{K}), B \in L(\mathcal{G}, \mathcal{K})$ and $R(B) \subseteq R(A)$, then $A^{\dagger} B$ is the Douglas solution of $A X=B$. 
Remark 2.3. If $R(A)$ is not closed, then $A^{\dagger}$ is a well defined unbounded operator and the result above is still valid. We leave the details for the reader.

Corollary 2.4. Under the same hypotheses of Theorem 2.1, every solution $\tilde{X}$ of $A X=B$ can be written as

$$
\tilde{X}=X_{0}+X_{\mathcal{M}}
$$

where $X_{0}$ is any solution of the homogeneous equation $A X=0$ and $X_{\mathcal{M}}$ is the reduced solution for some $\mathcal{M}$ as before.

The next result shows the relationship between different reduced solutions by means of certain oblique projections.

Theorem 2.5. Let $A \in C R(\mathcal{H}, \mathcal{K}), B \in L(\mathcal{G}, \mathcal{K})$ be such that $R(B) \subseteq R(A)$ and let $\mathcal{M}$ be a closed subspace of $\mathcal{H}$ such that $\mathcal{M}+N(A)=\mathcal{H}$. Then $X_{\mathcal{M}}$ is the reduced solution for $\mathcal{M}$ of the equation $A X=B$ if and only if $X_{\mathcal{M}}=Q_{\mathcal{M} / / N(A)} X_{R\left(A^{*}\right)}$.

Proof. Let $X_{\mathcal{M}}$ be the reduced solution for $\mathcal{M}$ of the equation $A X=B$. Then, as was shown in the proof of Theorem 2.1, there exists $Q^{\prime} \in \mathcal{Q}_{R(A)}$ such that $A^{\prime}=$ $Q_{\mathcal{M} / / N(A)} A^{\dagger} Q^{\prime} \in A[1,2]$ and $X_{\mathcal{M}}=A^{\prime} B$. Thus, $X_{\mathcal{M}}=A^{\prime} B=Q_{\mathcal{M} / / N(A)} A^{\dagger} Q^{\prime} B=$ $Q_{\mathcal{M} / N(A)} A^{\dagger} B=Q_{\mathcal{M} / / N(A)} X_{R\left(A^{*}\right)}$. The third equality holds because $R(B) \subseteq$ $R\left(Q^{\prime}\right)$.

Conversely, let $\hat{X}=Q_{\mathcal{M} / / N(A)} X_{R\left(A^{*}\right)}$. Since $A\left(X_{R\left(A^{*}\right)}-\hat{X}\right)=A\left(I-Q_{\mathcal{M} / / N(A)}\right)$ $X_{R\left(A^{*}\right)}=A Q_{N(A) / / \mathcal{M}} X_{R\left(A^{*}\right)}=0$ then $A \hat{X}=B$ and so $\hat{X}=X_{\mathcal{M}}$.

\section{Generalized inverses and Douglas equation}

In this section we study the equations $A X=Q$, where $Q$ is a projection onto the range of $A$ and the relationship between their solutions with the sets $A[1], A[1, i]$, $A[1, i, j]$ and $A[1, i, j, k]$. Part of the information contained in the following result can be found disseminated in the literature. The reader is referred to the book by Ben-Israel and Greville [1] (see, in particular, chapter 1 and 2).

Theorem 3.1. Given $A \in C R(\mathcal{H}, \mathcal{K})$ it holds that:

(i) $A[1]=\left\{X \in L(\mathcal{K}, \mathcal{H}): A X=Q\right.$ for some $\left.Q \in \mathcal{Q}_{R(A)}\right\}$.

(ii) $A[1,2]=\left\{X \in L(\mathcal{K}, \mathcal{H}): A X=Q \in \mathcal{Q}_{R(A)}\right.$ and $\left.N(X)=N(Q)\right\}$.

(iii) $A[1,3]=\left\{X \in L(\mathcal{K}, \mathcal{H}): A X=P_{R(A)}\right\}$.

(iv) $A[1,4]=\left\{X \in L(\mathcal{K}, \mathcal{H}): X A=P_{R\left(A^{*}\right)}\right\}$.

(v) $A[1,2,3]=\left\{X \in L(\mathcal{K}, \mathcal{H}): A X=P_{R(A)}\right.$ and $\left.N(X)=R(A)^{\perp}\right\}$.

(vi) $A[1,2,4]=\left\{X \in L(\mathcal{K}, \mathcal{H}): X A=P_{R\left(A^{*}\right)}\right.$ and $\left.R(X) \subseteq R\left(A^{*}\right)\right\}$.

(vii) $A[1,3,4]=\left\{X \in L(\mathcal{K}, \mathcal{H}): A X=P_{R(A)}\right.$ and $\left.X A=P_{R\left(A^{*}\right)}\right\}$.

(viii) $A[1,2,3,4]=\left\{\right.$ Douglas solution of $\left.A X=P_{R(A)}\right\}$.

Proof.

(i) Let $X \in A[1]$, i.e., $X$ verifies that $A X A=A$. Hence, $A X A X=A X$ and $R(A) \subseteq R(A X) \subseteq R(A)$. Then $A X \in \mathcal{Q}_{R(A)}$. Conversely, let $X$ be such that $A X=Q$ for some $Q \in \mathcal{Q}_{R(A)}$, then $A X A=Q A=A$.

(ii) Consider $X \in A[1,2]$. Then, by (i), $A X=Q \in \mathcal{Q}_{R(A)}$ and so $N(X) \subseteq$ $N(Q)$. On the other hand, since $X=X A X=X Q, N(Q) \subseteq N(X)$. Thus, $N(X)=N(Q)$. On the contrary, choose $X \in L(\mathcal{K}, \mathcal{H})$ such that $A X=Q \in$ $\mathcal{Q}_{R(A)}$ and $N(X)=N(Q)$; then, by (i), $X \in A[1]$. In order to prove that $X \in A[1,2]$, it is sufficient to observe that $X A X=X Q=X$, where the last equality holds because $N(Q)=R(I-Q)=N(X)$. 
(iii) The proof is immediate: by (i), any $X \in A[1,3]$ satisfies $A X=Q$ for some $Q \in \mathcal{Q}_{R(A)}$ and $(A X)^{*}=A X$; then, $Q^{*}=Q$, and it must be $Q=P_{R(A)}$. The converse is obvious.

(iv) Let $X \in A[1,4]$; then $A^{*} X^{*} A^{*}=A^{*}$ and so $A^{*} X^{*} \in \mathcal{Q}_{R\left(A^{*}\right)}$. Now, since $X \in A[4], A^{*} X^{*}=X A=P_{R\left(A^{*}\right)}$. Conversely, if $X A=P_{R\left(A^{*}\right)}=A^{*} X^{*}$, then $A^{*} X^{*} A^{*}=A^{*}$ and so $X \in A[1,4]$.

(v) Let $X \in A[1,2,3]$; then, by (ii), $A X=Q \in \mathcal{Q}_{R(A)}$ and $N(X)=N(Q)$. Furthermore, since $X \in A[3], Q^{*}=(A X)^{*}=A X=Q$, so $Q=P_{R(A)}$ and $N(X)=R(A)^{\perp}$. To prove the converse, we take $X$ such that $A X=P_{R(A)}$ and $N(X)=R(A)^{\perp}$; then, by (ii), $X \in A[1,2]$ and $A X=(A X)^{*}$. This proves that $X \in A[1,2,3]$.

(vi) Let $X \in A[1,2,4]$. By (iv), $X A=P_{R\left(A^{*}\right)}$. Then $X=X A X=P_{R\left(A^{*}\right)} X$ and so $R(X) \subseteq R\left(A^{*}\right)=N(A)^{\perp}$. Conversely, by (iv), it only remains to show that $X \in A[2]$. Now, $X A X=P_{R\left(A^{*}\right)} X=X$ where the last equality holds because $R(X) \subseteq R\left(A^{*}\right)$.

(vii) It follows by (iii) and (iv).

(viii) It is sufficient to show that $A^{\dagger}$ is the Douglas reduced solution of $A X=$ $P_{R(A)}$, but this is immediate by (iii) and (vi).

Remark 3.2. If $A \in C R(\mathcal{H}, \mathcal{K})$, then the equation $A X=P_{R(A)}$ has a solution. Moreover, the Douglas solution is the Moore-Penrose inverse of $A$. This is the meaning of the last item of the theorem above. Therefore, necessary and sufficient conditions for $D \in L(\mathcal{K}, \mathcal{H})$ to be the Moore-Penrose inverse of $A$ are $A D=P_{R(A)}$ and $R(D) \subseteq N(A)^{\perp}$. This seems to be one of the shortest ways for testing MoorePenrose identities.

Remark 3.3. Of course, equation $X A=B$ is equivalent to $A^{*} X^{*}=B^{*}$. Therefore, the sets $A^{*}[1], A^{*}[1, i], A^{*}[1, i, j]$ and $A^{*}[1, i, j, k]$ are related to the solutions of the equations $X A=Q$, where $Q^{*} \in \mathcal{Q}_{R\left(A^{*}\right)}$.

Proposition 3.4. Let $A \in C R(\mathcal{H}, \mathcal{K})$ and $A^{\prime} \in L(\mathcal{K}, \mathcal{H})$. Then, $A^{\prime} \in A[1,2]$ if and only if $A^{\prime}=Q A^{\dagger} \tilde{Q}$, where $1-Q \in \mathcal{Q}_{N(A)}$ and $\tilde{Q} \in \mathcal{Q}_{R(A)}$.

Proof. Let $A^{\prime} \in A[1,2]$. Since $A A^{\dagger} A=A$ then $A^{\prime} A A^{\dagger} A A^{\prime}=A^{\prime}$, and the assertion follows taking $Q=A^{\prime} A$ and $\tilde{Q}=A A^{\prime}$. Observe that $Q=Q_{R\left(A^{\prime}\right) / / N(A)}$. In fact, $R(Q)=R\left(A^{\prime}\right)$ and $N(Q)=R\left(Q^{*}\right)^{\perp}=R\left(A^{*}\right)^{\perp}=N(A)$. The converse follows just by checking that $Q A^{\dagger} \tilde{Q} \in A[1,2]$.

Remark 3.5. Let $A \in C R(\mathcal{H}, \mathcal{K})$ and $\mathcal{X}_{R\left(A^{*}\right)}=\{X \in L(\mathcal{K}, \mathcal{H}): A X=Q$ for some $Q \in \mathcal{Q}_{R(A)}$ such that $\left.R(X) \subseteq R\left(A^{*}\right)\right\}$, i.e., $\mathcal{X}_{R\left(A^{*}\right)}$ is the set of Douglas solutions of the equations $A X=Q \in \mathcal{Q}_{R(A)}$. Observe that $\mathcal{X}_{R\left(A^{*}\right)} \subsetneq A[1,2]$. In fact, $\mathcal{X}_{R\left(A^{*}\right)}=$ $\left\{A^{\dagger} Q: Q \in \mathcal{Q}_{R(A)}\right\}$ and by the last proposition, $A[1,2]=\left\{\tilde{Q} A^{\dagger} Q: 1-\tilde{Q} \in\right.$ $\mathcal{Q}_{N(A)}$ and $\left.Q \in \mathcal{Q}_{R(A)}\right\}$. Then, for every $Q \in \mathcal{Q}_{R(A)}$ it holds that

$$
A^{\dagger} Q=P_{N(A)^{\perp}} A^{\dagger} Q \in A[1,2],
$$

which proves the inclusion. To see that it is a strict inclusion we present the following example. In what follows, for a fixed $A \in C R(\mathcal{H}, \mathcal{K})$ we use the $2 \times 2$ matrix representation of operators $\mathcal{H} \longrightarrow \mathcal{K}$ induced by the decompositions $\mathcal{H}=$ 
$R\left(A^{*}\right) \oplus N(A)$ and $\mathcal{K}=R(A) \oplus N\left(A^{*}\right)$. According to these decompositions

$$
A=\left(\begin{array}{cc}
a & 0 \\
0 & 0
\end{array}\right) \text {, }
$$

observe that $a: R\left(A^{*}\right) \rightarrow R(A)$ is an isomorphism. Now, let $x \in L\left(R\left(A^{*}\right), N(A)\right)$, $x \neq 0$. Define $\tilde{Q}=\left(\begin{array}{ll}1 & 0 \\ x & 0\end{array}\right) \in L(\mathcal{H})$ and $Q=\left(\begin{array}{ll}1 & 0 \\ 0 & 0\end{array}\right) \in L(\mathcal{K})$. Then $\tilde{Q} A^{\dagger} Q=\left(\begin{array}{cc}a^{-1} & 0 \\ x a^{-1} & 0\end{array}\right) \in A[1,2]$ and $R\left(\tilde{Q} A^{\dagger} Q\right)=R\left(A^{*}\right) \oplus R(x) \nsubseteq R\left(A^{*}\right)$. Therefore $\tilde{Q} A^{\dagger} Q \notin \mathcal{X}_{R\left(A^{*}\right)}$.

\section{About the Continuity of Reduced solutions}

It is well known that the mapping $A \rightarrow A^{\dagger}$ is not continuous, in general. See [8] for several results in these matters. We show here how the relationship between the Douglas solution and generalized inverses gives some continuity results for the mapping $(A, B) \rightarrow$ Douglas solution of $A X=B$.

In this section, for the sake of simplicity we only consider operators $\mathcal{H} \rightarrow \mathcal{H}$. For $T \in L(\mathcal{H})$ the reduced minimum modulus of $T$ is $\gamma(T)=\inf \left\{\|T x\|: x \in N(T)^{\perp}\right.$ and $\|x\|=1\}$. It is well known that $\gamma(T)>0$ if and only if $R(T)$ is closed.

For each positive integer $k$, let

$$
\mathcal{R}_{k}=\mathcal{R}_{k}(\mathcal{H})=\{T \in L(\mathcal{H}): \gamma(T) \geq 1 / k\} .
$$

These sets verify:

(1) $C R(\mathcal{H})=\bigcup_{k \in \mathbb{N}} \mathcal{R}_{k}$.

(2) $\mathcal{R}_{k} \subsetneq \mathcal{R}_{k^{\prime}}$ if $k^{\prime}>k$.

(3) $T \in \mathcal{R}_{k}$ if and only if $T^{*} \in \mathcal{R}_{k}$.

(4) For every $k \in \mathbb{N}, \mathcal{R}_{k}$ is closed.

Given $A \in C R(\mathcal{H})$ we define $\mathcal{E}_{A}=\{B \in L(\mathcal{H}): R(B) \subseteq R(A)\}$. Similarly, given $B \in L(\mathcal{H})$ we define $\mathcal{E}_{k}^{B}=\left\{A \in \mathcal{R}_{k}: R(B) \subseteq R(A)\right\}$. Consider the mappings $\phi_{A}: \mathcal{E}_{A} \rightarrow L(\mathcal{H})$ and $\phi_{k}^{B}: \mathcal{E}_{k}^{B} \rightarrow L(\mathcal{H})$ defined by $\phi_{A}(B)=\phi_{k}^{B}(A)=$ Douglas solution of the equation $A X=B$. Next, we study the continuity of these mappings.

Proposition 4.1. $\phi_{A}$ is continuous.

Proof. Let $B, \tilde{B} \in \mathcal{E}_{A}$. Then $\phi_{A}(B)=A^{\dagger} B$ and $\phi_{A}(\tilde{B})=A^{\dagger} \tilde{B}$. Let $\epsilon>0$. Then

$$
\left\|\phi_{A}(B)-\phi_{A}(\tilde{B})\right\|=\left\|A^{\dagger} B-A^{\dagger} \tilde{B}\right\|=\left\|A^{\dagger}(B-\tilde{B})\right\| \leq\left\|A^{\dagger}\right\|\|B-\tilde{B}\|<\epsilon
$$

if $\|B-\tilde{B}\|<\delta<\frac{\epsilon}{\left\|A^{\top}\right\|}$. Therefore, $\phi_{A}$ is continuous.

The next lemma will be used to prove the continuity of $\phi_{k}^{B}$. A proof of this result can be found in [2, Lemma 3.10.

Lemma 4.2. For every $A, B \in \mathcal{R}_{k}$ it holds that $\left\|A^{\dagger}-B^{\dagger}\right\| \leq 3 k^{2}\|A-B\|$. In particular, the map $\mu: \mathcal{R}_{k} \rightarrow C R(\mathcal{H})$ such that $\mu(C)=C^{\dagger}$ is Lipschitz.

Proposition 4.3. The mapping $\phi_{k}^{B}$ is continuous.

Proof. Let $\epsilon>0$ and $A, \tilde{A} \in \mathcal{E}_{k}^{B}$. Then $\phi_{k}^{B}(A)=A^{\dagger} B$ and $\phi_{k}^{B}(\tilde{A})=\tilde{A}^{\dagger} B$. Now, by Lemma 4.2 it holds that

$$
\begin{aligned}
\left\|\phi_{k}^{B}(A)-\phi_{k}^{B}(\tilde{A})\right\| & =\left\|A^{\dagger} B-\tilde{A}^{\dagger} B\right\|=\left\|\left(A^{\dagger}-\tilde{A}^{\dagger}\right) B\right\| \\
& \leq\left\|A^{\dagger}-\tilde{A}^{\dagger}\right\|\|B\| \leq 3 k^{2}\|A-\tilde{A}\|\|B\|<\epsilon
\end{aligned}
$$


if $\|A-\tilde{A}\|<\delta<\frac{\epsilon}{3 k^{2}\|B\|}$. Thus, $\phi_{k}^{B}$ is continuous.

Remark 4.4. If $\mathcal{E}^{B}=\{A \in C R(\mathcal{H}): R(B) \subseteq R(A)\}$, then $\phi^{B}: \mathcal{E}^{B} \rightarrow L(\mathcal{H})$ is not continuous in general. Indeed, $A \rightarrow A^{\dagger} B$ may fail to be continuous if there is no control on $\gamma(A)$.

Remark 4.5. Proposition 4.1 is still true if for each $\mathcal{M}$ such that $\mathcal{H}=N(A)+\mathcal{M}$, we consider the map $\phi_{A, \mathcal{M}}: \mathcal{E}_{A} \longrightarrow L(\mathcal{H})$ defined by $\phi_{A, \mathcal{M}}(B)=X_{\mathcal{M}}$, where $X_{\mathcal{M}}$ denotes the reduced solution for $\mathcal{M}$ of $A X=B$. In fact, $X_{\mathcal{M}}=A^{\prime} B$ for some $A^{\prime} \in A[1,2]$; then using the arguments of Proposition 4.1 the assertion follows.

\section{REFERENCES}

1. A. Ben-Israel and T. N. E. Greville, Generalized inverses. Theory and applications. Second edition, Springer-Verlag, New York, 2003. MR.1987382 (2004b:15008)

2. G. Corach, A. Maestripieri and M. Mbekhta, Metric and homogeneous structure of closed range operators, J. Operator Theory (in press).

3. F. Deutsch, The angle between subspaces of a Hilbert space, Approximation theory, wavelets and applications (S. P. Singh, ed.), Kluwer, Dordrecht, 1995, 107-130. MR1340886(96e:46027)

4. R. G. Douglas, On majorization, factorization and range inclusion of operators on Hilbert space, Proc. Amer. Math. Soc. 17 (1966) 413-416. MR0203464 (34:3315)

5. P. A. Fillmore and J. P. Williams, On operator ranges, Advances in Mathematics 7 (1971), 254-281. MR0293441(45:2518)

6. R. Kadison and J.R. Ringrose, Fundamentals of the theory of operator algebras. Vol. II, Graduate Studies in Mathematics, Amer. Math. Soc., Providence, RI, 1997. MR1468230 (98f:46001b)

7. T. Kato, Perturbation theory for linear operators (second edition), Springer-Verlag, BerlinNew York, 1976. MR0407617 (53:11389)

8. J.-Ph. Labrousse and M. Mbekhta, Les opérateurs points de continuité pour la conorme et l'inverse de Moore-Penrose, Houston J. Math. 18 (1992), 7-23. MR1159435 (93g:47004)

9. E. H. Moore, On the reciprocal of the general algebraic matrix, Bull. Amer. Math. Soc. 26 (1920), 394-395.

10. M. Z. Nashed, Inner, outer, and generalized inverses in Banach and Hilbert spaces, Numer. Funct. Anal. Optim. 9 (1987), 261-325. MR887072 (88g:47006)

11. R. A. Penrose, A generalized inverse for matrices, Proc. Cambridge Philos. Soc. 51 (1955), 406-413. MR0069793 (16:1082a)

12. R. A. Penrose, On best approximation solutions of linear matrix equations, Proc. Cambridge Philos. Soc. 52 (1956), 17-19. MR0074092(17:536d)

Instituto Argentino de Matematica, Saavedra 15, 1083 Buenos Aires, Argentina

E-mail address: ml_arias@uolsinectis.com.ar

Instituto Argentino de Matemática, SaAvedra 15, 1083 Buenos Aires, Argentina

E-mail address: gcorach@fi.uba.ar

Instituto Argentino de Matemática, SaAvedra 15, 1083 Buenos Aires, Argentina

E-mail address: celegonzalez@gmail.com 